PROCEEDINGS OF THE

AMERICAN MATHEMATICAL SOCIETY

Volume 129, Number 10, Pages 2947-2953

S 0002-9939(01)06064-6

Article electronically published on April 17, 2001

\title{
CONDITIONAL WEAK COMPACTNESS IN VECTOR-VALUED FUNCTION SPACES
}

\author{
MARIAN NOWAK
}

(Communicated by Dale Alspach)

\begin{abstract}
Let $E$ be an ideal of $L^{0}$ over a $\sigma$-finite measure space $(\Omega, \Sigma, \mu)$ and let $E^{\prime}$ be the Köthe dual of $E$ with $\operatorname{supp} E^{\prime}=\Omega$. Let $\left(X,\|\cdot\|_{X}\right)$ be a real Banach space, and $X^{*}$ the topological dual of $X$. Let $E(X)$ be a subspace of the space $L^{0}(X)$ of equivalence classes of strongly measurable functions $f: \Omega \rightarrow X$ and consisting of all those $f \in L^{0}(X)$ for which the scalar function $\|f(\cdot)\|_{X}$ belongs to $E$. For a subset $H$ of $E(X)$ for which the set $\left\{\|f(\cdot)\|_{X}: f \in H\right\}$ is $\sigma\left(E, E^{\prime}\right)$-bounded the following statement is equivalent to conditional $\sigma\left(E(X), E^{\prime}\left(X^{*}\right)\right)$-compactness: the set $\left\{\|f(\cdot)\|_{X}: f \in H\right\}$ is conditionally $\sigma\left(E, E^{\prime}\right)$-compact and $\left\{\int_{A} f(\omega) d \mu: f \in H\right\}$ is a conditionally weakly compact subset of $X$ for each $A \in \Sigma, \mu(A)<\infty$ with $\chi_{A} \in E^{\prime}$. Applications to Orlicz-Bochner spaces are given.
\end{abstract}

\section{INTRODUCTION AND PRELIMINARIES}

Given a dual pair $\langle L, K\rangle$, a subset $A$ of $L$ is said to be conditionally $\sigma(L, K)$ compact whenever each sequence in $A$ contains a $\sigma(L, K)$-Cauchy subsequence (cf. [MN, p. 100]). The problem of characterizing relatively sequentially $\sigma\left(L^{p}(X)\right.$, $L^{q}\left(X^{*}\right)$ )-compact subsets of Lebesgue-Bochner spaces $L^{p}(X)$ (where $1 \leqslant p<\infty$ and $q$ conjugate to $p$ ) over a finite measure space was considered by F. Bombal $\mathrm{B}_{1}$ and J. Batt and W. Hiermeyer [BH, Theorem 2.1]. Moreover, F. Bombal characterized relatively sequentially $\sigma\left(L^{\varphi}(X), L^{\varphi^{*}}\left(X^{*}\right)\right)$-compact subsets of Orlicz-Bochner spaces $L^{\varphi}(X) \mathrm{B}_{2}$, Theorem 3]. C. Abott, E. Bator, R. Bilyeu and P. Lewis [ABBL] obtained the following characterization of conditionally $\sigma\left(L^{1}(X), L^{\infty}\left(X^{*}\right)\right)$-compact subsets of $L^{1}(X)$.

Theorem 1.1 (cf. ABBL, Theorem 2.5]). Let $(\Omega, \Sigma, \mu)$ be a finite measure space. Then for a norm bounded subset $H$ of $L^{1}(X)$ the following statements are equivalent:

(i) $H$ is conditionally $\sigma\left(L^{1}(X), L^{\infty}\left(X^{*}\right)\right)$-compact.

(ii) a) The subset $\left\{\|f(\cdot)\|_{X}: f \in H\right\}$ of $L^{1}$ is uniformly integrable.

b) The set $\left\{\int_{A} f(\omega) d \mu: f \in H\right\}$ is conditionally weakly compact in $X$ for each $A \in \Sigma$.

In this paper, by making use of Theorem 1.1 we characterize conditionally $\sigma\left(E(X), E^{\prime}\left(X^{*}\right)\right)$-compact subsets of $E(X)$, where $E$ is an ideal of $L^{0}$ over a $\sigma$ finite measure space.

Received by the editors July 6, 1998 and, in revised form, February 14, 2000.

2000 Mathematics Subject Classification. Primary 46B25, 46 E40.

Key words and phrases. Conditional weak compactness, vector valued function spaces. 
Now we establish notation and terminology (see $[\mathrm{AB}],[\mathrm{KA}]$ ).

Let $(\Omega, \Sigma, \mu)$ be a complete $\sigma$-finite measure space and let $L^{0}$ denote the space of equivalence classes of all $\Sigma$-measurable functions defined and finite a.e. on $\Omega$. Let $\chi_{A}$ stand for the characteristic function of a set $A$ and let $\mathbb{N}$ denote the set of all natural numbers. Let $E$ be an ideal of $L^{0}$ with $\operatorname{supp} E=\Omega$, and let $E^{\prime}$ stand for the Köthe dual of $E$, i.e.,

$$
E^{\prime}=\left\{v \in L^{0}: \int_{\Omega}|u(\omega) v(\omega)| d \mu<\infty \quad \text { for all } u \in E\right\} .
$$

We assume that $\operatorname{supp} E^{\prime}=\Omega$.

Let $\left(X,\|\cdot\|_{X}\right)$ be a real Banach space, and let $S_{X}$ and $B_{X}$ denote the unit sphere and the closed unit ball in $X$, resp. Let $X^{*}$ stand for the Banach dual of $X$. By $L^{0}(X)$ we denote the set of equivalence classes of all strongly $\Sigma$-measurable functions $f: \Omega \rightarrow X$. For $f \in L^{0}(X)$ let us set $\widetilde{f}(\omega)=\|f(\omega)\|_{X}$ for $\omega \in \Omega$. Let

$$
E(X)=\left\{f \in L^{0}(X): \tilde{f} \in E\right\} .
$$

By $\sigma\left(E(X), E^{\prime}\left(X^{*}\right)\right)$ we will denote the weak topology on $E(X)$ with respect to the dual system $\left\langle E(X), E^{\prime}\left(X^{*}\right)\right\rangle$ under the natural duality $\langle f, g\rangle=\int_{\Omega}\langle f(\omega), g(\omega)\rangle d \mu$ for $f \in E(X), g \in E^{\prime}\left(X^{*}\right)$.

The following characterization of conditional $\sigma\left(E, E^{\prime}\right)$-compactness is needed.

Proposition $1.2\left(\left[\mathbb{N}_{2}\right.\right.$ Theorem 1.1]). For a $\sigma\left(E, E^{\prime}\right)$-bounded subset $A$ of $E$ the following statements are equivalent:

(i) $A$ is conditionally $\sigma\left(E, E^{\prime}\right)$-compact.

(ii) For each $v \in E^{\prime}$ the subset $\{u v: u \in A\}$ of $L^{1}$ is uniformly integrable.

(iii) The functional $p_{A}$ on $E^{\prime}$ defined by $p_{A}(v)=\sup _{u \in A} \int_{\Omega}|u(\omega) v(\omega)| d \mu$ is an order continuous Riesz seminorm.

\section{Conditionally $\sigma\left(E(X), E^{\prime}\left(X^{*}\right)\right)$-Compact sets in $E(X)$}

Let ca $(\Omega, \Sigma)$ stand for the Riesz space of countably additive set functions $\nu$ on $\Sigma$. For a sequence $\left(A_{n}\right)$ in $\Sigma$ we write $A_{n} \searrow_{\mu} \emptyset$ whenever $A_{n} \downarrow$ and $\mu\left(\bigcap_{n=1}^{\infty} A_{n}\right)=0$ (that is, $A_{n} \downarrow$ and $\mu\left(A_{n} \cap A\right) \rightarrow 0$ for each $A \in \Sigma$ with $\mu(A)<\infty$ ).

The following well-known result characterizes uniformly $\mu$-continuous sets in $\operatorname{ca}(\Omega, \Sigma)$.

Lemma 2.1. For a subset $\mathcal{K}$ of $c a(\Omega, \Sigma)^{+}$the following statements are equivalent:

(i) $\mathcal{K}$ is uniformly $\mu$-continuous (i.e., $\lim _{n}\left(\sup _{\nu \in \mathcal{K}} \nu\left(A_{n}\right)\right)=0$ as $A_{n} \searrow_{\mu} \emptyset$ ).

(ii) For each $\eta>0$ there exist $\delta>0$ and $A_{0} \in \Sigma$ with $\mu\left(A_{0}\right)<\infty$ such that $\nu(A) \leqslant \eta$ and $\nu\left(\Omega \backslash A_{0}\right) \leqslant \eta$ for all $A \in \Sigma$ with $\mu(A) \leqslant \delta$ and all $\nu \in \mathcal{K}$.

We shall need the following technical result.

Proposition 2.2. Let $\mathcal{K}$ be a subset of $c a(\Omega, \Sigma)^{+}$such that each $\nu \in \mathcal{K}$ is $\mu$ continuous. Assume that $\mathcal{K}$ is not uniformly $\mu$-continuous. Then there exist a pairwise disjoint sequence $\left(B_{n}\right)$ in $\Sigma$, a number $\varepsilon_{0}>0$ and a sequence $\left(\nu_{n}\right)$ in $\mathcal{K}$ such that $\nu_{n}\left(B_{n}\right)>\varepsilon_{0}$ for all $n \in \mathbb{N}$. 
Proof. In view of Lemma 2.1 there exists $\varepsilon_{0}>0$ such that either there exist a sequence $\left(A_{n}\right)$ in $\Sigma$ and a sequence $\left(\nu_{n}^{1}\right)$ in $\mathcal{K}$ such that

$$
\mu\left(A_{n}\right) \rightarrow 0 \quad \text { and } \quad \nu_{n}^{1}\left(A_{n}\right)>2 \varepsilon_{0}
$$

or there exists a sequence $\left(\nu_{n}^{2}\right)$ in $\mathcal{K}$ such that

$$
\nu_{n}^{2}\left(\Omega \backslash \Omega_{n}\right)>2 \varepsilon_{0}
$$

whenever $\Omega_{n} \uparrow \Omega$ and $\mu\left(\Omega_{n}\right)<\infty$ for $n \in \mathbb{N}$.

Assume that condition (1) holds. Then arguing as in [BL, p. 546] one can find a pairwise disjoint sequence $\left(B_{n}\right)$ in $\Sigma$ and a subsequence $\left(\nu_{k_{n}}^{1}\right)$ of $\left(\nu_{n}^{1}\right)$ such that $\nu_{k_{n}}^{1}\left(B_{n}\right) \geqslant \varepsilon_{0}$. Let $\nu_{n}=\nu_{k_{n}}^{1}$ for $n \in \mathbb{N}$.

Now assume that condition (2) holds. Let $C_{n}=\Omega \backslash \Omega_{n}$ for $n \in \mathbb{N}$. Then $C_{n} \searrow_{\mu} \emptyset$, so $\nu\left(C_{n}\right) \rightarrow 0$ for each $\nu \in \mathcal{K}$. Let $l_{1}=1$ and choose $l_{2} \in \mathbb{N}$ such that $l_{2}>l_{1}, \quad \nu_{l_{1}}^{2}\left(C_{l_{2}}\right)<\varepsilon_{0}$. Then choose $l_{3} \in \mathbb{N}$ such that $l_{3}>l_{2}$ and $\nu_{l_{2}}\left(C_{l_{3}}\right)<\varepsilon_{0}$. Continuing this process inductively we can find an increasing sequence $\left(l_{n}\right)$ in $\mathbb{N}$ such that $\nu_{l_{n}}^{2}\left(C_{l_{n+1}}\right)<\varepsilon_{0}$. Let $B_{n}=\Omega_{l_{n+1}} \backslash \Omega_{l_{n}}$ for $n \in \mathbb{N}$. Then $\left(B_{n}\right)$ is a disjoint sequence and since $B_{n}=C_{l_{n}} \backslash C_{l_{n+1}}$ for $n \in \mathbb{N}$, by making use of (2) we obtain that $\nu_{l_{n}}^{2}\left(B_{n}\right)=\nu_{l_{n}}^{2}\left(C_{l_{n}}\right)-\nu_{l_{n}}^{2}\left(C_{l_{n+1}}\right)>2 \varepsilon_{0}-\varepsilon_{0}=\varepsilon_{0}$. Put $\nu_{n}=\nu_{l_{n}}^{2}$ for $n \in \mathbb{N}$.

For a subset $H$ of $E(X)$ let $\widetilde{H}=\{\tilde{f}: f \in H\}$.

Now we are ready to state our main result.

Theorem 2.3. Let $H$ be a subset of $E(X)$ such that the subset $\widetilde{H}$ of $E$ is $\sigma\left(E, E^{\prime}\right)$ bounded. Then the following statements are equivalent:

(i) $H$ is conditionally $\sigma\left(E(X), E^{\prime}\left(X^{*}\right)\right)$-compact.

(ii) a) $\widetilde{H}$ is conditionally $\sigma\left(E, E^{\prime}\right)$-compact.

b) $\left\{\int_{A} f(\omega) d \mu: f \in H\right\}$ is a conditionally weakly compact subset of $X$ for each $A \in \Sigma, \mu(A)<\infty$ with $\chi_{A} \in E^{\prime}$.

Proof. (i) $\Rightarrow$ (ii) To prove that (a) holds, in view of Proposition 1.2 it is enough to show that for each $0 \leqslant v \in E^{\prime}$ the subset $\{\tilde{f} v: f \in H\}$ of $L^{1}$ is uniformly integrable. Assume on the contrary that there exists $0 \leqslant v_{0} \in E^{\prime}$ such that the set $\left\{\widetilde{f} v_{0}: f \in\right.$ $H\}$ is not uniformly integrable. For each $f \in H$ set $\nu_{f}(A)=\int_{A} \widetilde{f}(\omega) v_{0}(\omega) d \mu$ for $A \in \Sigma$. Then $\nu_{f}$ is a non-negative $\mu$-continuous countably additive set function on $\Sigma$ but the family $\left\{\nu_{f}: f \in H\right\}$ is not uniformly $\mu$-continuous. Hence in view of Proposition 2.2 there exist a pairwise disjoint sequence $\left(B_{n}\right)$ in $\Sigma$, a sequence $\left(f_{n}\right)$ in $H$, and a number $\varepsilon_{0}>0$ such that $\nu_{f_{n}}\left(B_{n}\right)=\int_{B_{n}} \widetilde{f}_{n}(\omega) v_{0}(\omega) d \mu>\varepsilon_{0}$ for each $n \in \mathbb{N}$. Clearly $v_{0} f_{n} \in L^{1}(X)$, so in view of $[\mathrm{Bu}$, Theorem 1.1.(4)]

$$
\begin{aligned}
\nu_{f_{n}}\left(B_{n}\right) & =\left\|\chi_{B_{n}} v_{0} \widetilde{f}_{n}\right\|_{L^{1}}=\left\|\chi_{B_{n}} v_{0} f_{n}\right\|_{L^{1}(X)} \\
& =\sup \left\{\left|\int_{B_{n}}\left\langle v_{0}(\omega) f_{n}(\omega), g(\omega)\right\rangle d \mu\right|: g \in L^{\infty}\left(X^{*}\right),\|g\|_{L^{\infty}\left(X^{*}\right)} \leqslant 1\right\} .
\end{aligned}
$$

Hence one can produce a sequence $\left(g_{n}\right)$ in $L^{\infty}\left(X^{*}\right)$ with $\left\|g_{n}\right\|_{L^{\infty}\left(X^{*}\right)} \leqslant 1, \chi_{\Omega \backslash B_{n}} g_{n}$ $=0$ and such that

$$
\left|\int_{B_{n}}\left\langle v_{0}(\omega) f_{n}(\omega), g_{n}(\omega)\right\rangle d \mu\right|>\varepsilon_{0} .
$$


Set $g_{0}=\sum_{n=1}^{\infty} g_{n}$. Then $g_{0} \in L^{0}\left(X^{*}\right)$ and $\left\|g_{0}\right\|_{L^{\infty}\left(X^{*}\right)} \leqslant 1$. Clearly $v_{0} g_{0} \in$ $E^{\prime}\left(X^{*}\right)$, so $\chi_{A} v_{0} g_{0} \in E^{\prime}\left(X^{*}\right)$ for each $A \in \Sigma$. In view of the assumption (i) there exists a $\sigma\left(E(X), E^{\prime}\left(X^{*}\right)\right)$-Cauchy subsequence $\left(f_{k_{n}}\right)$ of $\left(f_{n}\right)$ so for each $A \in$ $\Sigma, \lim _{n} \int_{A}\left\langle f_{k_{n}}(\omega), v_{0}(\omega) g_{0}(\omega)\right\rangle d \mu$ exists. Setting $\mu_{n}(A)=\iint_{A}\left\langle f_{k_{n}}(\omega), v_{0}(\omega) g_{0}(\omega)\right\rangle d \mu$ for $A \in \Sigma$, in view of Nikodym's convergence theorem (see [D, Chap. 7]), $\left\{\mu_{n}: n \in\right.$ $\mathbb{N}\}$ is uniformly countably additive on $\Sigma$. Hence there exists $m_{0} \in \mathbb{N}$ such that for $m \geqslant m_{0}, \sup \left|\mu_{n}\left(B_{m}\right)\right| \leqslant \varepsilon_{0}$ (see [D] Chap. 7, Theorem 10]). Hence for each $m \geqslant m_{0}$ we get ${ }^{n}$

$$
\begin{aligned}
\left|\mu_{m}\left(B_{k_{m}}\right)\right| & =\left|\int_{B_{k_{m}}}\left\langle f_{k_{m}}(\omega), v_{0}(\omega) g_{k_{m}}(\omega)\right\rangle d \mu\right| \\
& =\left|\int_{B_{k_{m}}}\left\langle v_{0}(\omega) f_{k_{m}}(\omega), g_{k_{m}}(\omega)\right\rangle d \mu\right| \leqslant \varepsilon_{0}
\end{aligned}
$$

which contradicts (1). This contradiction establishes that (a) holds.

To show that (b) holds, take $A \in \Sigma$ with $\chi_{A} \in E^{\prime}$, and let $\left(f_{n}\right)$ be a sequence in $H$. Set $g=\chi_{A} x^{*}$ where $x^{*} \in S_{X^{*}}$. Then $g \in E^{\prime}\left(X^{*}\right)$ and by assumption (i) there exists a subsequence $\left(f_{k_{n}}\right)$ of $\left(f_{n}\right)$ such that $\lim _{n} \int_{\Omega}\left\langle f_{k_{n}}(\omega), g(\omega)\right\rangle d \mu$ exists. Since $\int_{\Omega}\left\langle f_{k_{n}}(\omega), g(\omega)\right\rangle d \mu=x^{*}\left(\int_{A} f_{k_{n}}(\omega) d \mu\right)$, the set $\left\{\int_{A} f(\omega) d \mu: f \in H\right\}$ is conditionally weakly compact in $X$.

(ii) $\Rightarrow$ (i) Let $\left(f_{n}\right)$ be a sequence in $H$. Since supp $E^{\prime}=\Omega$ there exists a sequence $\left(\Omega_{m}\right)$ in $\Sigma$ such that $\Omega_{m} \uparrow \Omega$ and $\mu\left(\Omega_{m}\right)<\infty, \chi_{\Omega_{m}} \in E^{\prime}$ for $m \in \mathbb{N}$ (see [Z, Theorem 86.2]). Setting $A_{m}=\Omega \backslash \Omega_{m}$ for $m \in \mathbb{N}$ we see that $A_{m} \searrow_{\mu} \emptyset$. Given $m \in \mathbb{N}$ we have $\sup _{n} \int_{\Omega_{m}} \widetilde{f}_{n}(\omega) d \mu=c_{m}<\infty$, because $\chi_{\Omega_{m}} \in E^{\prime}$ and $\widetilde{H}$ is $\sigma\left(E, E^{\prime}\right)$-bounded. Hence $\left\{\chi_{\Omega_{m}} f_{n}: n \in \mathbb{N}\right\} \subset L_{\Omega_{m}}^{1}(X)$, and by assumption (a), $\left\{\chi_{\Omega_{m}} \widetilde{f}_{n}: n \in \mathbb{N}\right\}$ is a uniformly integrable subset of $L_{\Omega_{m}}^{1}$. Combining this observation with (b), in view of Theorem 1.1 we see that $\left\{\chi_{\Omega_{m}} f_{n}: n \in \mathbb{N}\right\}$ is a conditionally $\sigma\left(L_{\Omega_{m}}^{1}(X), L_{\Omega_{m}}^{\infty}\left(X^{*}\right)\right)$-compact subset of $L_{\Omega_{m}}^{1}(X)$.

In view of the above observation there exists a $\sigma\left(L_{\Omega_{1}}^{1}(X), L_{\Omega_{1}}^{\infty}\left(X^{*}\right)\right)$-Cauchy subsequence $\left(\chi_{\Omega_{1}} f_{k_{n}^{1}}\right)$ of $\left(\chi_{\Omega_{1}} f_{n}\right)$. Next, there exists a $\sigma\left(L_{\Omega_{2}}^{1}(X), L_{\Omega_{2}}^{\infty}\left(X^{*}\right)\right)$-Cauchy subsequence $\left(\chi_{\Omega_{2}} f_{k_{n}^{2}}\right)$ of $\left(\chi_{\Omega_{1}} f_{k_{n}^{1}}\right)$. It follows that the diagonal sequence $\left(f_{k_{n}^{n}}\right)$ has the property that for each $m \in \mathbb{N}\left(\chi_{\Omega_{m}} f_{k_{n}^{n}}\right)$ is a $\sigma\left(L_{\Omega_{m}}^{1}(X), L_{\Omega_{m}}^{\infty}\left(X^{*}\right)\right)$-Cauchy sequence. Put $h_{n}=f_{k_{n}^{n}}$ for $n \in \mathbb{N}$.

Let $g \in E^{\prime}\left(X^{*}\right)$. For $n \in \mathbb{N}$ let us put

$$
g_{n}(\omega)= \begin{cases}g(\omega) & \text { if } \omega \in \Omega_{n} \text { and }\|g(\omega)\|_{X^{*}} \leqslant n \\ 0 & \text { elsewhere. }\end{cases}
$$

Given $\varepsilon>0$ there exist $m_{0} \in \mathbb{N}$ and $\delta>0$ such that

$$
\sup _{n} \int_{\Omega \backslash \Omega_{m_{0}}} \widetilde{f}_{n}(\omega) \widetilde{g}(\omega) d \mu \leqslant \frac{\varepsilon}{4} \text { and } \sup _{n} \int_{A} \widetilde{f}_{n}(\omega) \widetilde{g}(\omega) d \mu \leqslant \frac{\varepsilon}{4}
$$


for each $A \in \Sigma$ with $\mu(A) \leqslant \delta$. For $\eta=\frac{\varepsilon}{4 c_{m_{0}}}$ let

$$
B_{n}=\left\{\omega \in \Omega_{m_{0}}:\left\|g(\omega)-g_{n}(\omega)\right\|_{X^{*}} \geqslant \eta\right\} .
$$

It is easy to observe that $B_{n} \downarrow \emptyset$, so $\mu\left(B_{n}\right) \rightarrow 0$. Choose $n_{0} \in \mathbb{N}$ with $n_{0} \geqslant m_{0}$ such that $\mu\left(B_{n_{0}}\right) \leqslant \delta$. Then by $(2)$ we get

$$
\sup _{n} \int_{B_{n_{0}}} \widetilde{h}_{n}(\omega) \widetilde{g}(\omega) d \mu \leqslant \frac{\varepsilon}{4} .
$$

Hence, by (3) we have

$$
\begin{aligned}
& \left|\int_{\Omega_{m_{0}}}\left\langle h_{n}(\omega), g(\omega)-g_{n_{0}}(\omega)\right\rangle d \mu\right| \leqslant \int_{\Omega_{m_{0}}} \widetilde{h}_{n}(\omega)\left\|g(\omega)-g_{n_{0}}(\omega)\right\|_{X^{*}} d \mu \\
\leqslant & \int_{B_{n_{0}}} \widetilde{h}_{n}(\omega)\left\|g(\omega)-g_{n_{0}}(\omega)\right\|_{X^{*}} d \mu+\int_{\Omega_{m_{0}} \backslash B_{n_{0}}} \widetilde{h}_{n}(\omega)\left\|g(\omega)-g_{n_{0}}(\omega)\right\|_{X^{*}} d \mu \\
\leqslant & \int_{B_{n_{0}}} \widetilde{h}_{n}(\omega) \widetilde{g}(\omega) d \mu+\eta \int_{\Omega_{m_{0}}} \widetilde{h}_{n}(\omega) d \mu \leqslant \frac{\varepsilon}{4}+\frac{\varepsilon}{4 c_{m_{0}}} \cdot c_{m_{0}}=\frac{\varepsilon}{2} .
\end{aligned}
$$

Since $\int_{\Omega_{m_{0}}}\left\langle h_{n}(\omega), g_{n_{0}}(\omega)\right\rangle d \mu \underset{n}{\longrightarrow} a$ for some $a \in \mathbb{R}$, we can choose $n_{1} \in \mathbb{N}$ such that for $n \geqslant n_{1}$

$$
\left|\int_{\Omega_{m_{0}}}\left\langle h_{n}(\omega), g_{n_{0}}(\omega)\right\rangle d \mu-a\right| \leqslant \frac{\varepsilon}{4} .
$$

Thus by (2), (4) and (5) for $n \geqslant n_{1}$ we get

$$
\begin{aligned}
& \left|\int_{\Omega}\left\langle h_{n}(\omega), g(\omega)\right\rangle d \mu-a\right| \\
\leqslant & \left|\int_{\Omega \backslash \Omega_{m_{0}}}\left\langle h_{n}(\omega), g(\omega)\right\rangle d \mu\right|+\left|\int_{\Omega_{m_{0}}}\left\langle h_{n}(\omega), g(\omega)-g_{n_{0}}(\omega)\right\rangle d \mu\right| \\
& +\left|\int_{\Omega_{m_{0}}}\left\langle h_{n}(\omega), g_{n_{0}}(\omega)\right\rangle d \mu-a\right| \leqslant \frac{\varepsilon}{4}+\frac{\varepsilon}{2}+\frac{\varepsilon}{4}=\varepsilon .
\end{aligned}
$$

This shows that $\left(h_{n}\right)$ is a $\sigma\left(E(X), E^{\prime}\left(X^{*}\right)\right)$-Cauchy subsequence of $\left(f_{n}\right)$, so $H$ is conditionally $\sigma\left(E(X), E^{\prime}\left(X^{*}\right)\right)$-compact.

Corollary 2.4. Assume that a Banach space $X$ contains no isomorphic copy of $\ell^{1}$, and let $H$ be a subset of $E(X)$ such that $\widetilde{H}$ is $\sigma\left(E, E^{\prime}\right)$-bounded. Then the following statements are equivalent:

(i) $H$ is conditionally $\sigma\left(E(X), E^{\prime}\left(X^{*}\right)\right)$-compact.

(ii) $\widetilde{H}$ is conditionally $\sigma\left(E, E^{\prime}\right)$-compact.

Proof. (i) $\Rightarrow$ (ii) Obvious.

(ii) $\Rightarrow$ (i) In view of Theorem 2.3 it is enough to show that $\left\{\int_{A} f(\omega) d \mu: f \in H\right\}$ is a conditionally weakly compact subset of $X$ for each $A \in \Sigma$ such that $\chi_{A} \in$ $E^{\prime}$. In fact, let $A \in \Sigma, \mu(A)<\infty$ with $\chi_{A} \in E^{\prime}$. Hence $\sup _{f \in H}\left\|\int_{A} f(\omega) d \mu\right\| \leqslant$ 
$\sup _{f \in H} \int_{A} \tilde{f}(\omega) d \mu<\infty$, so in view of the Rosenthal's $\ell^{1}$-theorem [R] $\left\{\int_{A} f(\omega) d \mu: f \in\right.$ $H\}$ is a conditionally weakly compact subset of $X$, as desired.

Assume now that $\left(E,\|\cdot\|_{E}\right)$ is a Banach function space. Then the space $E(X)$ provided with the norm $\|f\|_{E(X)}:=\|\widetilde{f}\|_{E}$ is usually called a Köthe-Bochner space. The associated norm $\|\cdot\|_{E^{\prime}}$ on the Köthe dual $E^{\prime}$ can be defined as follows:

$$
\|v\|_{E^{\prime}}=\sup \left\{\left|\int_{\Omega} u(\omega) v(\omega) d \mu\right|: u \in E,\|u\|_{E} \leqslant 1\right\} .
$$

Clearly, for a subset $H$ of $E(X)$ the set $\widetilde{H}$ is $\sigma\left(E, E^{\prime}\right)$-bounded whenever

$$
\sup _{f \in H}\|f\|_{E(X)}<\infty .
$$

Combining Corollary 2.4 and Proposition 1.2 we get:

Corollary 2.5. Let $\left(E,\|\cdot\|_{E}\right)$ be a Banach function space, and assume that $X$ contains no isomorphic copy of $\ell^{1}$. Then the following statements are equivalent:

(i) The associated norm $\|\cdot\|_{E^{\prime}}$ on $E^{\prime}$ is order continuous.

(ii) Every norm bounded set in $E(X)$ is conditionally $\sigma\left(E(X), E^{\prime}\left(X^{*}\right)\right)$-compact.

We now apply the previous results to Orlicz spaces (see [KR], L for more details).

By a Young function we mean a mapping $\varphi:[0, \infty) \rightarrow[0, \infty)$ that is convex, vanishes only at 0 and $\lim _{t \rightarrow 0} \frac{\varphi(t)}{t}=0, \quad \lim _{t \rightarrow \infty} \frac{\varphi(t)}{t}=\infty$. Let $L^{\varphi}$ be the Orlicz space associated with $\varphi$ and provided with the Luxemburg norm $\|u\|_{\varphi}:=\inf \{\lambda>$ $\left.0: \int_{\Omega} \varphi(|u(\omega)| / \lambda) d \mu \leqslant 1\right\}$. Then $\left(L^{\varphi}\right)^{\prime}=L^{\varphi^{*}}$, where $\varphi^{*}$ denotes the complementary Young function.

We say that a Young function $\psi$ increases more rapidly than another $\varphi$, in symbols $\varphi \prec \psi$, if for each $c>0$ there is $d>1$ such that $c \varphi(t) \leqslant \frac{1}{d} \psi(d t)$ for all

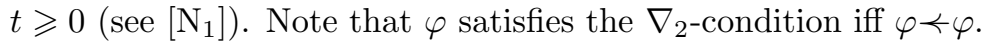

As a consequence of Corollary 2.4 and $\left[\mathrm{N}_{1}\right.$, Theorem 2.5] we get:

Corollary 2.6. Assume that $X$ contains no isomorphic copy of $\ell^{1}$. Then for a norm bounded subset $H$ of the Orlicz-Bochner space $L^{\varphi}(X)$ the following statements are equivalent:

(i) $H$ is conditionally $\sigma\left(L^{\varphi}(X), L^{\varphi^{*}}\left(X^{*}\right)\right)$-compact.

(ii) There is a Young function $\psi$ with $\varphi \prec \psi$ and such that $H \subset L^{\psi}(X)$ and $\sup _{f \in H}\|f\|_{L^{\psi}(X)}<\infty$.

Corollary 2.7. Assume that $X$ contains no isomorphic copy of $\ell^{1}$ and $\varphi$ satisfies the $\nabla_{2}$-condition. Then every norm bounded subset of $L^{\varphi}(X)$ is conditionally $\sigma\left(L^{\varphi}(X), L^{\varphi^{*}}\left(X^{*}\right)\right)$-compact.

\section{REFERENCES}

[AB] C.D. Aliprantis and O. Burkinshaw, Locally solid Riesz spaces, Academic Press, New York, San Francisco, London, 1978. MR 58:12271

[ABBL] C. Abbott, E. Bator, R. Bilyeu, P. Lewis, Weak precompactness, strong boundedness, and weak complete continuity, Math. Proc. Camb. Phil. Soc., 108 (1990), 325-335. MR 92b:46047 
[BH] J. Batt, W. Hiermeyer, On compactness in $L_{p}(\mu, X)$ in the weak topology and in the topology $\sigma\left(L_{p}(\mu, X), L_{q}\left(\mu, X^{\prime}\right)\right)$, Math. Z., 182 (1983), 409-423. MR 84m:46039

$\left[\mathrm{B}_{1}\right] \quad$ F. Bombal, On the space $L^{p}(\mu, X)$, Rev. Real Acad. Cienc. Exact. Fis. Natur. Madrid, 74, no. 1 (1980), 131-135 (in Spanish). MR 82i:46042

$\left[\mathrm{B}_{2}\right] \quad$ F. Bombal, On Orlicz space of vector-valued functions, Collect. Math., 32, no. 1 (1981), 3-12 (in Spanish). MR 83a:46039

[BL] R. Bilyeu, P. Lewis, Uniform differentiability, uniform absolute continuity and the VitaliHahn-Saks theorem, Rocky Mtn. J. Math., 10, No. 3 (1980), 533-557. MR 82g:46083

$[\mathrm{Bu}] \quad \mathrm{A} . \mathrm{V}$. Bukhvalov, On an analytic representation of operators with abstract norm, Izv. Vyss. Uceb. Zaved., 11 (1975), 21-32 (in Russian).

[DU] J. Diestel, J.J. Uhl Jr., Vector measures, Math. Surveys, 15, Amer. Math. Soc., Providence, R.I., 1977. MR 56:12216

[D] J. Diestel, Sequences and series in Banach spaces, Graduate Texts in Math., SpringerVerlag, New York, 1984. MR 85i:46020

[KA] L.V. Kantorovitch, A.V. Akilov, Functional analysis, Nauka, Moscow, 1984 ( $3^{\text {rd }}$ ed.) (in Russian).

[KR] M. Krasnoselskii, Ya. B. Rutickii, Convex functions and Orlicz spaces, P. Noordhoff Ltd, Groningen, 1961. MR 23:A4016

[L] W. Luxemburg, Banach function spaces, Delft, 1955. MR 17:285a

[MN] P. Mayer-Nieberg, Banach lattices, Springer-Verlag, Berlin, Heidelberg, New York, 1991.

$\left[\mathrm{N}_{1}\right] \quad$ M. Nowak, Order continuous seminorms and weak compactness in Orlicz spaces, Collect. Math., 44 (1993), 217-236. MR 95g:46055

$\left[\mathrm{N}_{2}\right] \quad$ M. Nowak, Weak sequential compactness in non-locally convex Orlicz spaces, Bull. Pol. Acad. Sci., 46 (1998), 225-231. MR 99g:46034

[R] H.P. Rosenthal, A characterization of Banach spaces containing $\ell^{1}$, Proc. Nat. Acad. Sci. U.S.A., 71 (1974), 2411-2413. MR 50:10773

[Z] A.C. Zaanem, Riesz spaces II, North Holland Pub. Comp., Amsterdam, New York, Oxford, 1983.

Institute of Mathematics, T. Kotarbiński Pedagogical University, Pl. SŁOwiański 9, 65-069 Zielona Góra, Poland

E-mail address: mnowa@lord.wsp.zgora.pl 